ARTÍCULOS ORIGINALES

Rev Chil Salud Pública 2015; Vol 19 (3): 261-269

Valentina Díaz ${ }^{1}$

Gloria Echagüe ${ }^{1}$ Malvina PÁez ${ }^{2}$

Laura Mendoza ${ }^{2}$

Pamela Mongelós ${ }^{2}$

Amalia Castro ${ }^{2}$

María ISABel Rodríguez ${ }^{2}$

Graciela Giménez ${ }^{1}$

Patricia Araujo ${ }^{2}$

Florentina Laspina ${ }^{3}$

Wilberto Castro ${ }^{4}$

Ramón Marecos ${ }^{5}$

Gerardo Deluca ${ }^{6}$

Alejandra Picconi ${ }^{7}$

ROSA JIMÉNEZ ${ }^{8}$

1 Departamento de

Bioquímica Clínica, Instituto de Investigaciones de las

Ciencias de la Salud, Campus Universitario,

San Lorenzo, Paraguay.

Departamento de Salud

Pública, Instituto de

Investigaciones de las

Ciencias de la Salud, Campus Universitario,

San Lorenzo, Paraguay.

${ }^{3}$ Departamento de

Microbiología, Instituto

de Investigaciones de las

Ciencias de la Salud, Campus Universitario,

San Lorenzo, Paraguay.

${ }^{4}$ Facultad de Ciencias Médicas,

Universidad Nacional de

Asunción, Asunción, Paraguay.

${ }_{5}^{5}$ Hospital Regional de Villa

Hayes, Ministerio de Salud

Pública y Bienestar

Social, Paraguay.

'Laboratorio de Aplicaciones

Moleculares. Cátedra de

Microbiología de la Universidad

Nacional del Nordeste,

Resistencia, Argentina.

Servicio Virus Oncogénicos, Instituto Nacional de Enfermedades Infecciosas (INEI)ANLIS, Dr. Malbrán,

Buenos Aires, Argentina.

${ }^{3}$ Departamento de Inmunología,

Instituto de Investigaciones de las Ciencias de la Salud,

Campus Universitario,

San Lorenzo, Paraguay.

nitadiazp@gmail.com

\section{ANEMIA Y DEFICIENCIA DE HIERRO EN MUJERES INDÍGENAS DEL DEPARTAMENTO DE PRESIDENTE HAYES, PARAGUAY, 2010-2011}

ANEMIA AND IRON DEFICIENCY IN INDIGENOUS WOMEN FROM THE Department of Presidente Hayes, Paraguay, 2010-2011

\section{RESUMEN}

Según datos de la UNICEF, entre 4 mil y 5 mil millones de personas en el mundo padecen carencia de hierro, y se calcula que 2 mil millones sufren de anemia. La mujer puede presentar diversos tipos de anemia, ya sea por menstruaciones abundantes o por deficiencia de aporte de hierro. El objetivo de este trabajo fue evaluar los parámetros hematológicos e indicadores quimicos de la anemia y carencia de hierro en las mujeres indígenas del Departamento de Presidente Hayes, Paraguay, realizado de octubre de 2010 a octubre de 2011. En este estudio observacional descriptivo de corte transverso, se incluyeron 222 mujeres de 12 a 60 años de edad de las etnias maká, nivaclé, sanapaná, enxet y tobaqom. Se determinó la concentración de la hemoglobina e indices hematimétricos: VCM, HCM y CHCM mediante un contador hematológico. El hierro sérico y transferrina se determinó por método enzimático colorimétrico y la saturación de la transferrina por cálculo manual. En el $40 \%$ (88/222) de las mujeres se observó hemoglobina por debajo de $12 \mathrm{~g} / \mathrm{dL}$. Con respecto a los indices hematimétricos, presentaron valores inferiores al punto de corte, siendo el $33 \%$ para VCM, 22\% HCM y el 16\% para CHCM. Estos resultados indican una deficiencia de bierro en diferentes fases, lo que tendría consecuencias negativas como la anemia, baja resistencia a infecciones, retraso en el desarrollo psicomotor y función cognoscitiva de los niños, bajo rendimiento académico, fatiga, entre otras patologías en una población vulnerable donde la mayoría de las mujeres estudiadas son de edad fértil.

Palabras clave: anemia, ferropenia, mujeres indígenas.

\begin{abstract}
According to UNICEF, between 4 thousand and 5 thousand million people in the world have ferropenia and it is calculated that 2 thousand million suffer from anemia. Women may present different types of anemia, due either to abundant menstruation or deficient iron intake. The objective of this work was to evaluate hematological and chemical parameters of anemia in indigenous women from the Department of Presidente Hayes, Paraguay, from October 2010 to October 2011. This was a cross-sectional, observational and descriptive study that included 222 women aged 12 to 60
\end{abstract}

Recibido 20 de mayo 2015, aceptado 17 de julio 2015 
years, belonging to the ethnic groups maká, nivaclé, sanapaná, enxet and tobaqom. Hemoglobin concentration and hematological parameters were determined using a bematological counter. Serum iron and transferrin were determined by a colorimetric enzymatic method, and transferrin saturation by manual calculation. In 40\% (88/222) of the women, bemoglobin values under $12 \mathrm{~g} / \mathrm{dL}$ were found. Hematological parameters were below established cut-off points in 33\% of women for $M C V, 22 \%$ for $M C H$ and $16 \%$ for $M C H C$. These results indicate iron deficiency in different stages, which could have negative consequences including anemia, low resistance to infections, retardation in psychomotor development and cognitive function in children, as well as low academic performance and other pathologies, affecting a vulnerable population where most studied women were of a reproductive age.

Keywords: anemia, ferropenia, indigenous women.

\section{INTRODUCCIÓN}

Según datos del Fondo de las Naciones Unidas para la Infancia (UNICEF), entre 4 mil y 5 mil millones de personas en el mundo padecen carencia de hierro, y se calcula que 2 mil millones sufren de anemia. Los sectores de la población más vulnerables son las mujeres y los niños de corta edad. El hierro se almacena en la médula ósea, el bazo y el hígado. La carencia de hierro se produce cuando se reducen de manera considerable esas reservas de hierro, mientras que la anemia ocurre al agotarse dichas reservas. ${ }^{1}$

En situaciones de volemia normal, la Organización Mundial de la Salud (OMS) define anemia como el descenso en la concentración de hemoglobina por debajo de $12 \mathrm{~g} / \mathrm{dL}$ en mujeres. La anemia ferropénica se caracteriza por el descenso en la concentración de hemoglobina tal y como se ha definido anteriormente y por un perfil férrico deficitario. Generalmente los glóbulos rojos son de menor tamaño (volumen corpuscular medio [VCM] inferior a $80 \mathrm{fL}$ ). Si existe una situación de anemia ferropénica o de ferropenia sin anemia, la valoración individualizada estará en función de la edad y el sexo. ${ }^{2}$ Se sabe que vivir a cierta altitud por encima del nivel del mar y el tabaquismo aumentan las concentraciones de hemoglobina. Además de éstos, se ha propuesto que hay pequeñas diferencias en las distribuciones de los valores de hemoglobina según los grupos étnicos. ${ }^{3}$

El consumo insuficiente de hierro es solo una de las causas de la carencia del mismo. La falta de hierro en el organismo puede producir mala síntesis proteica, deficiencia inmunitaria, menor compensación de enfermedades cardiopulmonares y anemia. Otros transtornos que pueden causar o agravar la carencia de hierro son las pérdidas de sangre durante la menstruación y las infecciones parasitarias, como el paludismo y los parásitos intestinales. ${ }^{1,4}$

La anemia aumenta el peligro de hemorragias y sepsis (infección bacteriana aguda) durante el parto y está relacionada con un $20 \%$ de las muertes maternas. El consumo insuficiente de hierro es solo una de las causas de la carencia del mismo. ${ }^{1}$

Los índices hematimétricos son los parámetros que relacionan hematocrito, hemoglobina y número de eritrocitos o hematíes. Éstos son el volumen corpuscular medio (VCM), la hemoglobina corpuscular media (HCM) y la concentración de la hemoglobina corpuscular media (CHCM).5,6

La falta de hierro es la causa más frecuente de anemia. Los valores normales de hierro en el organismo son de unos 50-55 mg por $\mathrm{kg}$ de peso en el hombre y de unos 35-40 mg por $\mathrm{kg}$ de peso en la mujer. La ingesta de hierro diaria suele ser de entre 10 y $30 \mathrm{mg}$, y la absorción ocurre principalmente en el duodeno. Una vez absorbido, pasa a la sangre unida en su mayor parte a una proteína transportado- 
ra, la transferrina, aunque una porción menor se une a otra proteína llamada ferritina (no evaluada en este trabajo), que permite valorar los depósitos de hierro del organismo. La anemia ferropénica puede ser debida a tres causas principalmente: un descenso del aporte de hierro en la dieta; una disminución de la absorción del hierro a nivel del aparato digestivo por diferentes causas; un aumento de las pérdidas de sangre, como pueden ser sangrados gastrointestinales crónicos, menstruaciones abundantes, otras pérdidas ginecológicas o bien el embarazo, dado que el feto utiliza dos tercios del hierro que absorbe la madre. ${ }^{7}$

El proceso de la deficiencia de hierro consta de tres estadios: 1) El primer estadio, denominado "Agotamiento de los depósitos de hierro", donde la ferritina inicia un proceso de disminución; 2) en el segundo estadio: "Eritropoyesis deficiente en hierro". En esta etapa el hierro sérico y la saturación de la transferrina presentan valores inferiores. 3) tercer estadio, denominado "Anemia microcítica hipocrómica”, disminuye la síntesis de la hemoglobina, y es el examen de laboratorio más utilizado para definir dicho estadio. ${ }^{8}$

Las pérdidas fisiológicas diarias de hierro en varones son alrededor de $1 \mathrm{mg} /$ día, por heces, sudor, orina o descamación de la piel; mientras que en mujeres en edad fértil es de 1,5 mg/día, debido a la menstruación. En cada menstruación normal las pérdidas son aproximadamente $20 \mathrm{mg}$ de hierro. La anemia ferropénica tiene como población diana a los niños, adolescentes, mujeres en edad fértil y ancianos. La Atención Primaria es el primer nivel de atención en donde podemos detectar la existencia de un déficit de hierro en los diferentes grupos poblacionales de riesgo. En el mundo, entre el $66 \%$ y el $80 \%$ de la población puede ser deficiente en hierro y más del 30\% presenta anemia ferropénica. Este hecho constituye un importante problema de salud pública ya que afecta a un gran número de personas; estudios realizados en las poblaciones de alto riesgo como niños y mujeres en edad fértil han demostrado que la prevalencia de la ferropenia oscila entre el $50 \%$ en países en desarrollo y el $10 \%$ en aquellos con programas de prevención es- tablecidos. La ferropenia es un déficit muy frecuente, que afecta al 11\% de las mujeres, preferentemente en edad fértil y al $4 \%$ de los varones. La anemia ferropénica se detecta en $1-2 \%$ de los adultos. ${ }^{2}$

Existen trabajos científicos que demuestran que los pueblos indígenas afrontan un mayor riesgo de inseguridad alimentaria $y$ malnutrición que otros grupos, ya que padecen niveles más elevados de pobreza, una menor disponibilidad de recursos y una creciente dependencia de alimentos más baratos aunque con un alto grado de procesamiento. ${ }^{9}$

Siendo el tema de la salud una demanda prioritariamente del sector femenino indígena, reclaman su derecho al ejercicio de una salud reproductiva plena y con respeto a la cosmovisión indígena. Las reivindicaciones políticas de las mujeres han planteado que sin equidad de género no se puede hablar de desarrollo pleno. Las poblaciones indígenas son víctimas de abusos sistemáticos. Son las mujeres las que mayormente experimentan violaciones a sus derechos humanos relacionados a su género: ultrajes, esterilizaciones forzadas, servicios inadecuados de salud y desprecio de su lengua tradicional. ${ }^{10}$

En México y Guatemala se ha identificado que el riesgo de muerte durante el embarazo es tres veces más alto para las mujeres indígenas que para las no indígenas. Por otra parte, que el $40 \%$ de las mujeres indígenas mexicanas sufre de anemia en relación a un $26.4 \%$ de la población nacional, en relación al 35\% de las mujeres indígenas guatemaltecas en edad fértil que sufre desnutrición. ${ }^{10}$

Entre los principales factores que condicionan la salud de las mujeres indígenas, se encuentran: su historia reproductiva (cantidad de hijos engendrados, tipos de embarazos y partos, etc.) y por otra, su historia productiva y laboral (trabajos pesados y condiciones de éstos). Además, las prácticas culturalmente aceptadas como el matrimonio precoz, y otras, derivan en aspectos como abandono temprano de la escuela, alto número de hijos, mayor exposición a la violencia de pareja, pocas posibilidades de trabajo asalariado, etcétera. La carencia de educación sexual, unida a tabúes y falta de diálogo de pareja en torno a 
la sexualidad en las comunidades indígenas, provoca que las mujeres tengan dificultad para espaciar los embarazos. Por ejemplo, las mujeres indígenas paraguayas tienen una tasa de fertilidad de 7.3 hijos por mujer, las mayas rurales de Guatemala 6 hijos, en relación a la media de la mujeres indígenas bolivianas cuya tasa es 4.6. Todas estas cifras anteriores difieren de la media latinoamericana de 2.7 hijos. ${ }^{10}$

En relación a la salud de las mujeres indígenas paraguayas, existe, por una parte, un deficiente acceso a los sistemas formales, como instituciones públicas, hospitales, y por otra, mantienen sus prácticas tradicionales como la asistencia por los chamanes, parteras empíricas y médicos naturalistas. $.^{10},{ }^{11}$ Un aspecto muy importante de la anemia en las mujeres, consiste en que aumenta de modo notable su riesgo de muerte durante o después del parto. La mujer puede sangrar abundantemente y tener reservas bajas de hemoglobina. ${ }^{12}$ En Paraguay se estima que 40 mujeres jóvenes mueren cada año durante el embarazo y el parto por anemia severa, causada por deficiencia de hierro. ${ }^{13}$

Este trabajo tiene por objetivo evaluar los parámetros hematológicos e indicadores químicos de la anemia y carencia de hierro en las mujeres indígenas del Departamento de Presidente Hayes, Paraguay.

\section{MATERIAL Y MÉTODO}

El presente trabajo es un estudio observacional descriptivo de corte transverso, realizado desde octubre de 2010 hasta octubre de 2011. Se incluyeron 222 mujeres indígenas de varias comunidades, de 12 a 60 años de edad, del Departamento de Presidente Hayes, Paraguay (Figura 1). El material fue tomado previo consentimiento informado escrito. Fueron incluidas en el estudio 42 mujeres de la comunidad de Quenkuket de la etnia Maká, 55 de la comunidad de Novotac (Marcelo Cué y Duarte Cué) de la etnia Nivaclé, 43 de la comunidad de Makxawaya, 20 de Espinillo, 20 de Pozo Colorado, de la etnia Enxet y 42 de la comunidad TobaQom de la etnia Toba Qom.
La extracción de sangre se realizó por punción venosa, con ayuno previo; el contenido de la jeringa $(5 \mathrm{ml})$ se distribuyó en tubos de polietileno glicol con $50 \mu \mathrm{l}$ de anticoagulante EDTA (etilendiamino tetra acetato $0,342 \mathrm{~mol}$ ) para 2,5 $\mathrm{ml}$ de sangre; $y$ otro en tubos secos para las determinaciones de hierro y transferrina.

La determinación de la concentración de la hemoglobina e índices hematimétricos, se realizó con el contador hematológico $\mathrm{ABX}$ Micros 60 (método de impedancia). El hierro sérico y transferrina se determinaron por método enzimático colorimétrico, en el autoanalizador WIENER lab: CB350i (Italia) y la saturación de la transferrina por cálculo manual. Se consideraron como puntos de corte: hemoglobina inferior a $12 \mathrm{~g} / \mathrm{dL}, \mathrm{VCM}$ menor a $80 \mathrm{fL}, \mathrm{HCM}$ por debajo de 27pg, CHCM inferior a $32 \mathrm{~g} / \mathrm{dL}$, hierro $(\mathrm{Fe})$ menor a $50 \mu \mathrm{g} /$ $\mathrm{dl}$, transferrina superior a $400 \mu / \mathrm{dL}$ y la saturación de la transferrina inferior a $20 \%,{ }^{1,2,14,15}$

También se aplicó una encuesta de forma individual para recoger algunos datos demográficos de interés como: la edad, nivel de escolaridad y hábitos alimentarios.

\section{Asuntos estadísticos}

El programa estadístico empleado para el análisis de los datos fue el Epi-info versión 3.5.3. (Atlanta, CDC). Con los datos obtenidos se creó una base de datos en planilla Excel para su análisis. Se realizó un análisis descriptivo mediante distribuciones de frecuencias para las variables cuali y cuantitativas. Se utilizó la prueba estadística chi cuadrado para comparar las proporciones de los resultados, considerándose con significancia estadística cuando el valor de p era menor a 0,05.

Las comunidades más cercanas fueron las de Quenkunquet, Río Verde y Toba Qom; como se puede observar en el mapa, éstas se encuentran en las proximidades de Villa $\mathrm{Ha}$ yes. En tanto que las más lejanas: las comunidades de Marcelo Cué y Duarte Cué del complejo Novoctas quedan a $170 \mathrm{~km}$ de camino de tierra de Pozo Colorado por la ruta Gral. Díaz. El complejo Laguna Pato se encuentra a $70 \mathrm{~km}$ de Pozo Colorado por un camino privado. 
Figura 1. Localización de las distintas comunidades indígenas del Departamento de Presidente Hayes, Chaco paraguayo.

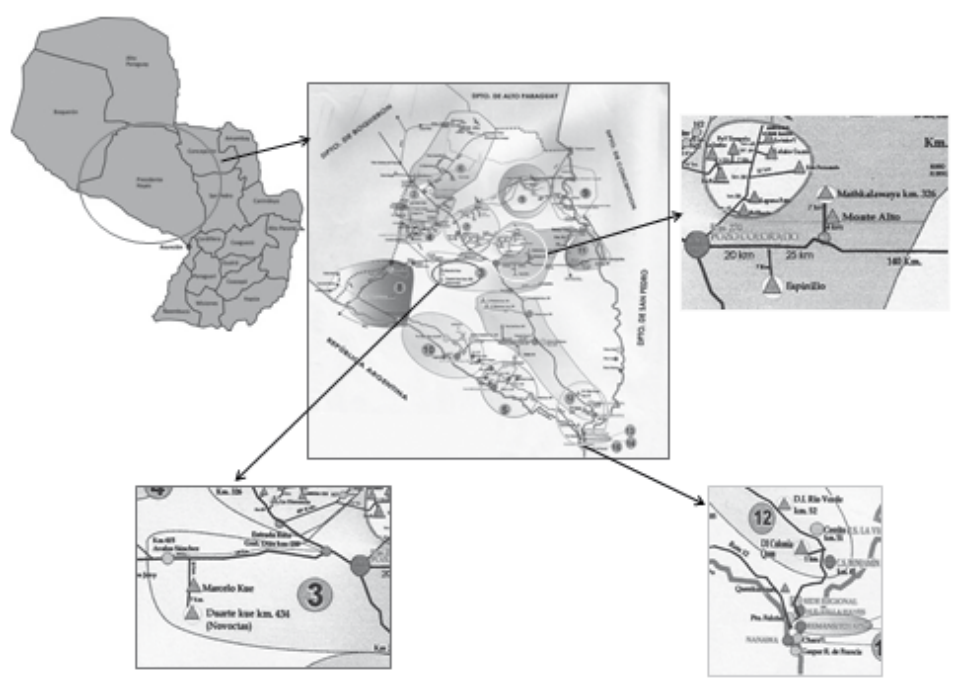

\section{Consideraciones éticas}

Fueron respetados los principios éticos de autonomía, respeto y justicia. Antes de la realización del estudio, las mujeres participantes firmaron un consentimiento informado aprobado por el Comité de Ética del Instituto de Investigaciones de la Salud de la Universidad Nacional de Asunción, y los representantes indígenas acompañaron y fueron mediadores en todo el proceso. En el caso de las mujeres indígenas que hablaban dialectos, el consentimiento informado fue traducido a su idioma y explicado por miembros de su comunidad, al cual daban su conformidad mediante la firma o impresión digital; y los padres o tutores de los menores de 18 años autorizaron la participación de los mismos. Todos los estudios realizados en forma gratuita, fueron entregados al Hospital Regional de Villa Hayes, situado en el área de influencia de las localidades estudiadas. Dicho hospital estatal tiene el compromiso de realizar el seguimiento y tratamiento de las personas afectadas según su diagnóstico final.

\section{RESULTADOS}

La población estudiada incluyó a mujeres entre 12 y 65 años de edad, con una media de 31 \pm 12 años. En el 39,6\% (88/222) de las mujeres se observaron valores de hemoglobina por debajo de $12 \mathrm{~g} / \mathrm{dL}$. Con respecto a los índices hematimétricos, el 32,8\% (73/222) presentó valores de VCM inferiores a $80 \mathrm{fL}$; para el HCM, 21,6\% (84/222) menor a 27 pg y el $15,7 \%$ (35/222) menor a $32 \mathrm{~g} / \mathrm{dL}$ para CHCM.

En relación al hierro, el $20 \%(44 / 222)$ tenía valores menores a $50 \mu \mathrm{g} / \mathrm{dL}$; y el $38,3 \%$ $(50 / 222)$ la transferrina superior a $400 \mu \mathrm{g} / \mathrm{dl}$. En cuanto a la saturación de la transferrina, el $47,3 \%(105 / 222)$ presentó valores por debajo de $20 \%$.

La población estudiada fue clasificada por edad, mostrando que todos los grupos etarios presentaron valores por debajo del punto de corte para la hemoglobina, el hierro, la saturación de la transferrina, el VCM, HCM y CHCM; en tanto que para la transferrina fue superior a dicho punto, como se puede observar en la Tabla 1. 
Tabla 1. Parámetros hematológicos según franja etaria

\begin{tabular}{|c|c|c|c|c|c|c|c|c|c|c|c|c|c|c|}
\hline \multirow[t]{2}{*}{ Edad } & \multicolumn{2}{|c|}{$\begin{array}{c}\text { Hierro } \\
<50 \mu / d L\end{array}$} & \multicolumn{2}{|c|}{$\begin{array}{l}\text { Transferrina } \\
>400 \mu / d L\end{array}$} & \multicolumn{2}{|c|}{$\begin{array}{l}\text { Sat.Transf. } \\
<20 \%\end{array}$} & \multicolumn{2}{|c|}{$\begin{array}{l}\text { Hemoglobina } \\
<12 \mathrm{~g} / \mathrm{dL}\end{array}$} & \multicolumn{2}{|c|}{$\begin{array}{l}\text { VCM } \\
<80 f L\end{array}$} & \multicolumn{2}{|c|}{$\begin{array}{l}\text { HCM } \\
<27 \mathrm{pg}\end{array}$} & \multicolumn{2}{|c|}{$\begin{array}{l}\mathrm{CHCM} \\
<32 \mathrm{~g} / \mathrm{dL}\end{array}$} \\
\hline & $\mathrm{n}$ & $\%$ & $\mathrm{n}$ & $\%$ & $\mathrm{n}$ & $\%$ & $\mathrm{n}$ & $\%$ & $\mathrm{n}$ & $\%$ & $\mathrm{n}$ & $\%$ & $\mathrm{n}$ & $\%$ \\
\hline $12-23$ & 18 & 25 & 13 & 18 & 37 & 51 & 26 & 36 & 28 & 39 & 30 & 42 & 12 & 17 \\
\hline $24-34$ & 8 & 11 & 19 & 26 & 32 & 44 & 28 & 39 & 21 & 29 & 24 & 33 & 11 & 15 \\
\hline $35-45$ & 9 & 21 & 12 & 28 & 17 & 40 & 16 & 37 & 11 & 26 & 16 & 37 & 8 & 19 \\
\hline $46-65$ & 9 & 26 & 6 & 17 & 19 & 54 & 18 & 51 & 13 & 37 & 14 & 40 & 4 & 11 \\
\hline
\end{tabular}

En las comunidades de Brillante, Duarte Cué, Marcelo Cué, Espinillo, Laguna Pato, Monte Alto y Maxhaguaya (poblaciones más alejadas de la zona urbana), presentaron va- lores inferiores a los puntos de corte para la hemoglobina, hierro y CHCM y superiores para transferrina, con una diferencia significativa de $\mathrm{p}<0.05$, como se observa en la Tabla 2.

Tabla 2. Parámetros hematológicos según ubicación de las comunidades

\begin{tabular}{lccccc}
\hline & \multicolumn{2}{c}{ Comunidad CC } & \multicolumn{2}{c}{ Comunidad CL } & P* \\
\hline Hierro $<50 \mu / \mathrm{dL}$ & 12 & $(27 \%)$ & 32 & $(73 \%)$ & 0,004 \\
Transferrina $>400 \mu / \mathrm{dL}$ & 32 & $(64 \%)$ & 18 & $(36 \%)$ & 0,001 \\
Saturación de la transferrina $<20 \mu / \mathrm{dL}$ & 46 & $(44 \%)$ & 59 & $(56 \%)$ & 0,36 \\
Hemoglobina $<12 \mathrm{~g} / \mathrm{dL}$ & 30 & $(34 \%)$ & 58 & $(66 \%)$ & 0,004 \\
& 29 & $(40 \%)$ & 44 & $(60 \%)$ & 0,13 \\
HCM $<27 \mathrm{pg}$ & 38 & $(45 \%)$ & 46 & $(55 \%)$ & 0,48 \\
$\mathrm{CHCM}<32 \mathrm{~g} / \mathrm{dL}$ & 22 & $(63 \%)$ & 13 & $(37 \%)$ & 0,01 \\
\hline
\end{tabular}

$\mathrm{p}^{*}=$ potencia

$\mathrm{CC}=$ comunidades más cercana a la zona urbana: Quenkunquet, Pozo Colorado, Río Verde y Toba Qom

$\mathrm{CL}=$ comunidades alejadas de la zona urbana: Marcelo Cué, Duarte Cué, Maxhalaguaya, Espinillo, Laguna Pato, Brillante y Monte Alto.

\section{DISCUSIÓN}

La carencia de hierro es el problema nutricional más prevalente de los seres humanos. La causa habitual de la anemia es la carencia de hierro, aunque no necesariamente una carencia de consumo absoluto alimentario. ${ }^{13}$ En este trabajo realizado en mujeres indígenas pertenecientes a las etnias Maká, Nivaclé, Enxet y Toba Qom, los resultados muestran un alto porcentaje de anemia en las diferentes franjas etarias. Este resultado coincide con un estudio realizado en la etnia Yucpa del Amazonas venezolano, donde se observó anemia en las mujeres indígenas de todas las edades. ${ }^{16}$
Igualmente, en otro estudio realizado en ese país con la etnia Piaroa, se encontró anemia y deficiencia de hierro más alto en las mujeres de todas las edades, incluyendo mujeres mayores a 40 años de edad, con una prevalencia del $80 \% .^{17}$

También se encontró prevalencia de anemia en un trabajo realizado en mujeres tarahumara de México, donde la deficiencia de hierro fue la responsable de la mayoría de los casos de anemia..$^{18}$ En nuestro país, en un trabajo realizado entre 2001-2002, en población indígena de ambos sexos de la etnia Nivaclé del chaco paraguayo, se encontró un 47,8\% de anemia en las mujeres en la franja etaria de 20 a 40 años. ${ }^{19}$ 
Las enormes desigualdades que existen con respecto a los pueblos indígenas se ven reflejadas en los indicadores socioeconómicos, de salud y demográficos. A modo de ejemplo mencionamos la tasa bruta de mortalidad general, que en la población paraguaya no indígena es de 6,1 por mil habitantes mientras que en la población indígena es de un promedio de 16,9 por mil habitantes. La población indígena del Paraguay, afectada por una serie persistente de problemas, constituye el segmento poblacional más empobrecido entre los más pobres del país. No cuentan con una asistencia adecuada en salud, solo el $26 \%$ de las comunidades tiene acceso a un puesto o centro de salud. La mortalidad infantil alcanza al 93,9 por mil. El analfabetismo llega a un 94,1\% de la población de más de 15 años. ${ }^{20,21}$

La casi nula cobertura sanitaria a las comunidades indígenas (el 93\% de las viviendas no cuenta con disposición sanitaria ni agua potable), ha deteriorado aún más sus condiciones de vida. La ausencia de una asistencia activa a los casos infecciosos, la poca accesibilidad por causas geográficas, económicas, lingüísticas o culturales de los indígenas a los centros asistenciales, la falta de seguimiento ambulatorio a los pacientes indígenas y el frecuente abandono de los tratamientos, favorecen la permanencia de enfermedades en esta población. Otro grave problema es la deficiencia del sistema de información en salud, que no contempla entre sus datos una diferenciación e identificación étnica de los casos registrados ni de la ubicación territorial del paciente indígena. Esto dificulta la vigilancia de los eventos que los afectan y por ende la construcción de un perfil epidemiológico, lo que genera dificultades para la intervención oportuna y la sistematización de acciones dirigidas a la atención de salud de los pueblos indígenas. ${ }^{20}$

Según los resultados del Tercer Censo Nacional de Población y Viviendas para Pueblos Indígenas 2012, en el Paraguay existen actualmente 493 comunidades y 218 aldeas o barrios, que totalizan 711 comunidades. En cuanto a la situación de tierra de los pueblos indígenas, de las 493 comunidades, 357 declararon que poseen tierra propia. Además, del total de comunidades indígenas censadas, 254 declararon contar con alguna dificultad que afecta a los recursos naturales dentro de su territorio. ${ }^{22}$

Considerando que la tierra/territorio es la base para el desarrollo étnico y económico, se puede afirmar que la condición básica para la sobrevivencia de casi la mitad de las comunidades indígenas del Paraguay no está garantizada. ${ }^{21}$

No se encontraron trabajos con estudios de deficiencia de hierro en mujeres indígenas de Paraguay. Sin embargo, es importante detectar en sus primeros estadios, para ser tratados a tiempo, porque cuando aparece la anemia ya se ha pasado por todas las etapas de la deficiencia de hierro. ${ }^{5}$

Los hallazgos de este estudio indican la importancia de la implementación de programas para mejorar el consumo de hierro en la dieta, y acciones para el control de infecciones parasitarias, para reducir la pérdida sanguínea que puede ser consecuencia de estas mismas enfermedades.

Es muy importante continuar con las investigaciones sobre la deficiencia de hierro y anemia, debido a que pueden ser indicadores de una enfermedad subyacente y potencialmente curable si es diagnosticada precozmente. Este trabajo realizado en una población vulnerable como la que constituyen las mujeres indígenas, es un aporte valioso a la salud pública, para que se puedan realizar campañas informativas y de prevención en las poblaciones de riesgo, con el objeto de minimizar las repercusiones sociales, laborales y en definitiva mejorar los estándares de calidad de vida.

\section{AGRADECIMIENTOS}

A la técnica Stella Mari Vázquez, a los docentes investigadores del Departamento de Bioquímica Clínica del Instituto de Investigaciones de Ciencias de la Salud, a los profesionales de la XV Región Sanitaria del Departamento de Presidente Hayes y del Hospital Regional de Villa Hayes, a las mujeres y a los líderes indígenas, que hicieron posible este trabajo. Un especial agradecimiento a la Dra. María Teresa Cuevas por su asesoramiento en el área hematológica. 


\section{REFERENCIAS}

1. UNICEF. Nutrición: Hierro [en línea], 2002 [consultado 16.01.2014]. Disponible en: http://www.unicef.org/spanish/nutrition/23964_iron.html

2. Abós Olivares E, Cortés Villuendas MT, Franco García E, García López S, Giraldo Castellano P, Giralt Raichs M. Guía clinica de actuación diagnóstica y terapéutica en la anemia ferropénica [en línea]. Zaragoza, 2004 [consultado 19.11.2013]. Disponible en: http://www.fehha.org/pub/publicaciones/docs/guia_AF.pdf

3. Organización Mundial de la Salud. Concentraciones de hemoglobina para diagnosticar la anemia y evaluar su gravedad [en línea]. Ginebra: OMS, 2011 [consultado 20.07.2015]. Disponible en: http://www. who.int/vmnis/indicators/haemoglobin es.pdf

4. Agostini CV. Metabolismo del hierro [en línea] En: Primer Curso mediterráneo multidisciplinar en anemia ferropénica. Milán: Pharmanutra, 2013; p. 9 [consultado febrero 2014 ]. Disponible en: http://www. deficitdehierro.com/img/pdf/11_1er\%20 Curso\%20Mediterraneo\%20Multidisciplinar\%20en\%20Anemia\%20Ferropenica\%202013.pdf

5. Angel G. Interpretación clínica del laboratorio. Cuarta edición. Buenos Aires: Panamericana, 1993.

6. Campuzano-Maya G. Del hemograma manual al hemograma de cuarta generación. Medicina E Laboratorio [en línea], 2007; 13: 511-50 [consultado 12.09.2013]. Disponible en: http://www.medigraphic.com/ pdfs/medlab/myl-2007/myl011-12b.pdf

7. Cañadas Bustos D. Enfermedades: Hematología: Anemia ferropénica [en línea] [consultado 2.02.2014]. Disponible en: http:// www.mapfre.com/salud/es/cinformativo/ anemias.shtml

8. Reboso Pérez JG. Indicadores bioquímicos de la deficiencia de hierro. Rev Cuba Aliment Nutr, 1997; 11(1): 64-7.

9. FAO. Politica de la FAO sobre pueblos indígenas y tribales [en línea]. Roma: FAO, 2012 [consultado 2.04.2014]. Disponible en: http://www.fao.org/docrep/019/i3064s/ i3064s.pdf

10. Calfio Montalva M, Velasco LF. Seminario Internacional de Pueblos Indígenas y Afrodescendientes de América Latina y el Caribe: información sociodemográfica para políticas y programas [en línea]. Santiago de Chile: CEPAL, 2005 [consultado 2.04.2014]. Disponible en: http://www.osarguatemala.org/ sites/default/files/docs/brechas.pdf

11. Seminario: Pueblos Indígenas y Derecho a la Salud. Ministerio de Salud Pública y Bienestar Social [en línea]. Asunción, 2009. [consultado 27.07.2015]. Disponible en: www.mspbs.gov.py/v2/buscador. php? \&page $=662$

12. FAO. Carencia de hierro y otras anemias nutricionales. Cap 13 [en línea]. En: $N u-$ trición bumana en el mundo en desarrollo. Roma: FAO;1997 [consultado 10.06.2013]. Disponible en: http://www.fao.org/docrep/006/w0073s/w0073s0h.htm

13. UNICEF. Déficit vitamínico y mineral. Informe valorativo del daño para Paraguay [en línea]. Nueva York: UNICEF [consultado 2.04.2014] Disponible en: http:// www.micronutrient.org/VMD/DARs/Paraguay.pdf

14. Wiener Laboratorio. Rosario-Argentina. Fer color AA. Rosario: Wiener Laboratorio, 2000.

15. Wiener Laboratorio. Rosario-Argentina. Fer color transferrina. Rosario: Wiener Laboratorio, 2000.

16. Diez-Ewald M, Torres-Guerra E, Lets I, Layrisse M, Vizcaíno G, Arteaga Vizcaíno M. Anemia en poblaciones indígenas del occidente de Venezuela. Investig Clínica, 1999; 40(3): 191-202.

17. García-Casal MN, Leets I, Bracho C, Hidalgo M, Bastidas G, Gómez A, Peña A, Pérez H. Prevalence of anemia and deficiencies of iron, folic acid and vitamin B 12 in an Indigenous community from the Venezuelan Amazon whit a high incidence of malaria. Arch Latinoam Nutr, 2008; 58(1): 12-6.

18. Monarrez-Espino J, Martinez H, Greiner T. Iron deficiency anemia in Tarahumara women of reproductive-age in Northern Mexico. Salud Pública Méx [en línea], 2001 Oct 
[consultado 2014 Nov 12]; 43(5):392-401. Disponible en: http://www.scielo.org.mx/ scielo.php?script $=$ sci_arttext\&pid=S0036$36342001000500002 \& \operatorname{lng}=$ es.

19. Díaz RV, Sosa L, Guillén R, Pistilli N, Páez M, Almirón M, et al. Prevalencia de anemia en varias comunidades de la etnia Nivaclé del Chaco paraguayo. Mem Inst Investig Cienc Salud, 2013; 9(1): 15-21.

20. Ortega Rolón S, Peralta R. Coordinación General. Indicadores de salud indígena. En: Política Nacional de Salud Indígena [en línea]. Asunción: UNICEF; Consejo Nacional de Pastoral Indígena; Ministerio de Salud Pública y Bienestar Social; 2012 [consultado 27.07.2015]. Disponible en: http://www. mspbs.gov.py/planificacion/wp-content/ uploads/2012/06/Politica-Nacional-de-Salud-Indigena1.pdf

21. Estrategia Indígena. Proyecto: Mejorando la conservación de la Biodiversidad y el manejo sostenible de la tierra en el bosque atlántico del Paraguay. ITAIPU. SEAM.PRODERS. GEF. Banco Mundial [en línea] [consultado 1.08.2015]. Disponible en: http://www.paraguaybio.com.py/documentos/7_Estrategia\%20Indigena.pdf

22. Dirección General de Estadística, Encuestas y Censos (Paraguay). Tercer Censo nacional de población y viviendas para pueblos indígenas: resultados preliminares 2012 [en línea]. Fernando de la Mora: DGEEC, 2012 [consultado 31.07.2015]. Disponible en: http://www.dgeec.gov.py/Publicaciones/ Biblioteca/censo\%20indigena \% 202012/ Pueblos\%20indigenas\%20en\%20el\%20Paraguay $\% 20 \% 20$ Resultados $\% 20$ preliminares\%20-\%20CNI\%202012.pdf

23. UNICEF; Organización Mundial de la Salud. Panamá: situación de deficiencia de hierro y anemia [en línea]. Panamá: UNICEF; 2006 [consultado 4.04.2014]. Disponible en: http://www.unicef.org/panama/spanish/ Hierro.pdf 